\title{
Behçet's Disease and Tuberculosis: A Complex Relationship
}

\author{
Sara Mendonça Freitas, Joana Silva Marques, Ana Grilo, Rodolfo Gomes, Fernando Martos Gonçalves \\ Serviço de Medicina Interna, Hospital Beatriz Ângelo, Loures, Portugal
}

Doi: 10.12890/2020_001354 - European Journal of Case Reports in Internal Medicine - C E EFIM 2020

Received: 30/10/2019

Accepted: 04/11/2019

Published: $16 / 01 / 2020$

How to cite this article: Mendonça Freitas S, Silva Marques J, Grilo A, Gomes R, Martos Gonçalves F. Behçet's disease and tubercolosis: a complex relationship. EJCRIM 2020;7: doi:10.12890/2020_001241.

Conflicts of Interests: The Authors declare that there are no competing interest

This article is licensed under a Commons Attribution Non-Commercial 4.0 License

\section{ABSTRACT}

Behçet's disease (BD) is a systemic vasculitis characterized by recurrent orogenital ulceration and several systemic manifestations (such as gastrointestinal involvement, vascular disease or arthritis). The pathogenesis is still unknown but the trigger role of certain pathogens such as Mycobacterium tuberculosis is well documented. Furthermore, patients with BD are more susceptible to tuberculosis due to immunity defects. Here, we describe the case of a 70-year-old woman with a history of recurrent oral aphthae and inflammatory arthritis presenting with extensive thrombosis of left upper limb major veins, a positive HLA B51 genotype and colon ulceration; hence, BD diagnosis was made after excluding other causes. Simultaneously, the patient had cutaneous abscesses not associated with immunosuppressive therapy with continuous development, and after recurrent negative tuberculosis work-up, M. tuberculosis was isolated in an abscess culture.

\section{LEARNING POINTS}

- Patients with Behçet's disease (BD), in the absence of anti-TNF-alpha therapy, have increased susceptibility to tuberculosis due to a defect in cell-mediated immunity.

- It is very important to distinguish between BD and pseudo-Behçet's at the onset of tuberculosis, since Behçet-like manifestations achieve complete remission with anti-bacillar therapy.

- Cutaneous tuberculosis is a rare condition, with a wide clinical spectrum; hence, high clinical suspicion, and sometimes, multiple bacteriological examinations, are required in order to diagnose.

\section{KEYWORDS}

Cutaneous tuberculosis, Behçet's disease, venous thrombosis

\section{CASE DESCRIPTION}

A 70-year-old woman, with a medical history of recurrent oral aphthae (more than 3 episodes per year) and recurrent inflammatory arthritis on the right shoulder, fists and ankles was admitted to the emergency department due to pain and oedema of the left upper limb, and a diagnosis of venous thrombosis of the cephalic, axillary, subclavian, internal jugular and brachiocephalic veins was made through a computed tomography (CT) scan. Inherited thrombophilia work-up was negative, anti-phospholipid antibodies were all negative, ANA were detected at a 1:640 titre, with a speckled pattern, and the HLA B51 allele was positive (Table 1). Thorax, abdomen and pelvis CT scanning showed no sign of cancer nor other abnormalities. Colonoscopy and upper endoscopy were also performed to rule out digestive cancer. At the ascending colon, there was an ulcerative lesion and biopsy showed a diffuse, eosinophilic inflammatory infiltrate, with a crypt abscess and ulceration. Mammary echography ruled out breast malignancy.

A month later, the patient developed lower right lobe pneumonia with pleural effusion and was treated with amoxicillin/clavulanate and azithromycin. Blood cultures and the Ziehl-Neelsen (ZN) smear test were negative. The pleural fluid was an exudate, ADA levels were high, although bacteriological, mycobacteriological cultures and ZN smear testing were negative. Bronchofibroscopy was performed and 
microbacteriological, mycobacteriological cultures, the ZN smear test and Mycobacterium tuberculosis DNA analysis of bronchoalveolar lavage fluid and bronchial secretions were negative. During hospitalization, the patient developed an erythematous papule on the left leg, that was excised. Once more, the bacteriological culture and ZN smear test were negative, and histology was compatible with an epidermoid cyst.

\begin{tabular}{|l|l|}
\hline Parameter & Result \\
\hline Factor V Leiden & negative \\
\hline Prothrombin mutation & negative \\
\hline Protein C & normal \\
\hline Protein S & normal \\
\hline Antithrombin III & normal \\
\hline ANA & 1 :640 (speckled pattern) \\
\hline Anti-dsDNA antibody & negative \\
\hline Anti-Sm antibody & negative \\
\hline Anti-RNP antibody & negative \\
\hline Anti-cardiolipin IgG and IgM antibodies & negative \\
\hline Beta-2 glycoprotein & negative \\
\hline Lupus anticoagulant & negative \\
\hline HLA B51 allele & positive \\
\hline Complement C3 & normal \\
\hline Complement C4 & slightly high \\
\hline Complement activity CH50 & normal \\
\hline Anti-SSA & negative \\
\hline Anti-SSB & negative \\
\hline Rheumatoid factor & negative \\
\hline Anti-CCP & negative \\
\hline HIV serology & negative \\
\hline HBV serology & negative \\
\hline HCV serology & negative \\
\hline
\end{tabular}

Table 1. Immunologic and serologic diagnostic tests. ANA: anti-neutrophil antibody, HLA: human leucocyte antigen, dsDNA: double-stranded DNA, RNP: ribonuclear protein, Sm: Smith, HIV: human immunodeficiency virus, HBV: hepatitis B virus, $\mathrm{HCV}$ : hepatitis C virus

After discharge, the clinical investigation proceeded and regarding the described clinical findings and a positive pathergy, a diagnosis of Behçet's disease (BD) was made. Therapy with prednisolone $20 \mathrm{mg} /$ day was initiated with complete disappearance of arthralgia and colon ulceration in a re-evaluation colonoscopy. The patient also had a tumefaction on the right chest wall before corticoid therapy was initiated. A CT scan showed some pulmonary fibrotic residual alterations, a collection in the right axillar area and right chest wall and several small lymph nodes at the anterior mediastinum and near the trachea. She was admitted again due to asthenia, abdominal pain, thoracic wall and inguinal tumefaction. At clinical examination, there were tumours at the axillary area, right hypochondrium with inflammatory signs and several inguinal nodules with skin fistulization. The TC abdominal scan showed an abscess on the right chest wall on cellular subcutaneous tissue (Fig. 1) and an ultrasound-guided abscess aspiration was performed. The bacteriologic culture and ZN smear test were negative but mycobacterial culture was positive for Mycobacterium tuberculosis at the 18th day of culture and the patient started treatment with isoniazid, rifampin, pyrazinamide and ethambutol with complete regression of cutaneous abscesses.

\section{DISCUSSION}

$\mathrm{BD}$ and cutaneous tuberculosis are 2 uncommon conditions and a simultaneous diagnosis is rare, especially in patients not receiving antiTNF-alpha therapy.

Tuberculosis and BD may have a common genetic background, and BD by itself may produce a defect in cell-mediated immunity, which increases the individual's susceptibility to tuberculosis ${ }^{[1]}$. Secondly, tuberculosis may play a key role in exacerbating BD, since there is evidence of infectious agents (including M. tuberculosis) triggering BD in patients with genetic susceptibility ${ }^{[2]}$. 


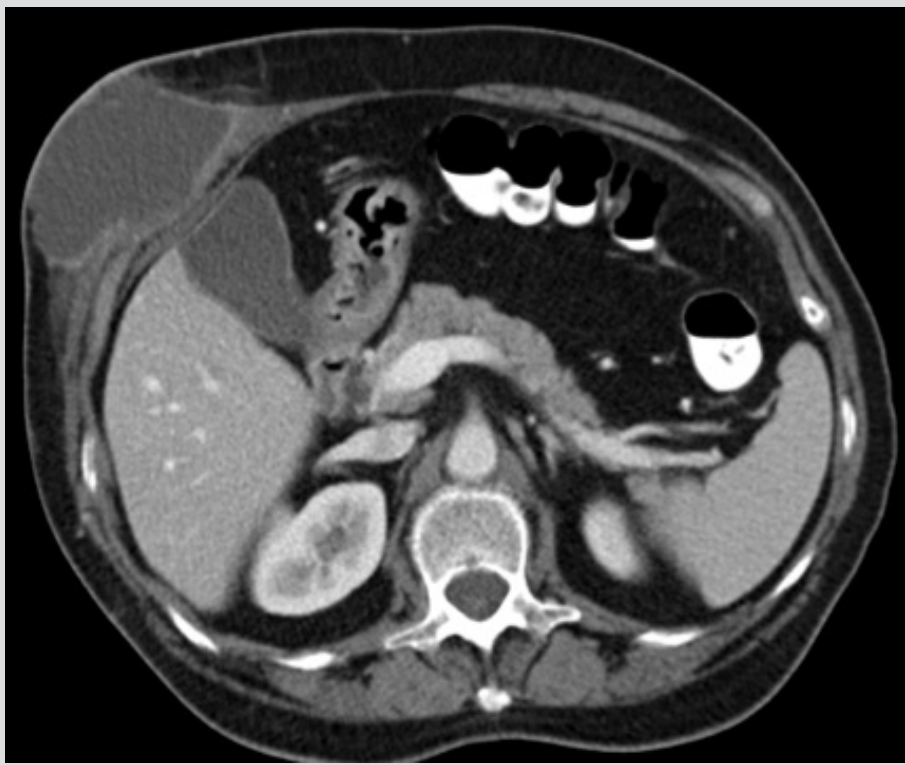

Figure 1. Mass in the subcutaneous tissue on the right chest wall with dimensions of $67 \times 90 \times 68 \mathrm{~mm}$, suggestive of inflammation/infection[

Furthermore, there is a condition known as pseudo-Behçet's that can mimic BD, consisting of hypersensitivity reactions to M. tuberculosis, such as reactive arthritis and erythema nodosum (Poncet's disease) and orogenital ulcers (as a result of cross-reactivity between microorganism antigens and mucosal proteins) ${ }^{[3]}$. The main difference is the complete response to anti-bacillar treatment. Behçet-like symptoms achieve complete remission with anti-bacillar therapy, unlike those associated with $\mathrm{BD}^{[1]}$.

In this case, considering: (i) positive HLA B51 genotyping that favours genetic susceptibility to BD, (ii) sufficient BD criteria according to The International Criteria for Behçet's Disease $2014^{[4]}$ (extensive venous thrombosis, history of recurrent oral ulcers and a positive pathergy test) and (iii) the clinical improvement of arthritis, oral ulcers and disappearance of colon ulceration with immunosuppressive therapy, we concluded the patient had BD exacerbated by cutaneous tuberculosis. In this particular case, immunosuppressive therapy did not play a role in triggering tuberculosis infection since the patient already had a cutaneous inflammatory tumefaction before prednisolone was initiated. Furthermore, neither venous thrombosis nor gastrointestinal involvement have been reported in pseudo-Behçet's, unlike what is seen in $\mathrm{BD}^{[1]}$.

Cutaneous tuberculosis is an uncommon type of tuberculosis and there is a wide spectrum of clinical manifestations. An abscess, sometimes with fistula formation, in certain body locations such as the chest wall, axillar and groin regions is highly typical of scrofuloderma ${ }^{[5]}$, and although a skin biopsy was not carried out this is the most probable diagnosis.

This report demonstrates the real challenge of BD diagnosis in a patient with skin tuberculosis regarding the complex aetiopathological relationship between those entities plus the difficult diagnosis of cutaneous tuberculosis by itself considering the wide clinical spectrum, and difficulties relating to bacteriological identification.

\section{REFERENCES}

1. Shinoda K, Hayashi R, Taki H, Hounoki H, Makino T, Nomoto K, et al. Pseudo-Behçet's disease associated with tuberculosis: a case report and review of the literature. Rheumatol Int 2014;34:1471-1474.

2. Cho S, Lee KJ, Lee JD, Bang D, Cho SB. Detection of tuberculous lymphadenopathy by positron emission tomography/computed tomography in a patient with Behçet's disease. Acta Derm Venereol 2011;91:470-471.

3. Mendes D, Correia M, Barbedo M, Vaio T, Mota M, Gonçalves O, et al. Behçet's disease-a contemporary review. J Autoimmunol 2009;32:178-188.

4. Davatchi F, Assaad-Khalil S, Calamia K, Crook J, Sadeghi-Abdollahi B, Schirmer M, et al. The International Criteria for Behçet's Disease (ICBD): a collaborative study of 27 countries on the sensitivity and specificity of the new criteria. J Eur Acad Dermatol Venereol 2014;28:338-347.

5. Bravo F, Gotuzzo E. Cutaneous tuberculosis. Clin Dermatol 2007;25:173-180. 